\title{
Pharmacokinetics of loop diuretics in congestive heart failure
}

\author{
D Craig Brater
}

Patients with congestive heart failure (CHF) represent one of the largest groups in which loop diuretics are a mainstay of treatment. Their proper use requires an understanding of the mechanisms of response to diuretics in such patients. Over the past few years information has increased concerning the pharmacokinetics and pharmacodynamics of loop diuretics in various diseases but particularly CHF. These data have in turn allowed a more rational design of therapeutic regimens. This review discusses our current understanding of mechanisms of resistance to loop diuretics in patients with CHF and shows how such understanding dictates therapeutic strategy.

\section{Bioavailability in patients and controls} As patients with CHF have peripheral oedema, it is not difficult to imagine how there might also be oedema of the intestinal wall that could impair absorption of loop diuretics and other drugs. ${ }^{12}$ This issue has been examined in several oedematous disorders but particularly in CHF. One case report found that a patient with idiopathic oedema seemed to malabsorb furosemide ${ }^{3}$; however, studies in $\mathrm{CHF}^{4-9}$ and in cirrhosis ${ }^{10}$ have indicated that bioavailability is normal in oedematous disorders. Importantly, this is the case in patients who have both compensated and decompensated $\mathrm{CHF}^{7}$ and in cirrhotic patients both with and without ascites. ${ }^{10}$ Thus malabsorption cannot be a mechanism for diuretic resistance in patients with $\mathrm{CHF}^{4-9}$

Detailed studies in patients with both compensated and decompensated CHF have shown that peak concentrations of loop diuretic are delayed and that concentrations are low in patients with CHF. ${ }^{5-7}$ Interestingly, a similar phenomenon occurs in patients with cirrhosis. ${ }^{10}$ As well as the delay in the time at which peak concentrations occur, there is also a decrease in the concentrations themselves. ${ }^{5-7}$ These findings imply that both the lag time for absorption is lengthened and that the rate of absorption is delayed (table). In patients with CHF, the more decompensated the patient the greater these abnormalities; in individual patients, as dry weight is attained absorption variables return toward but not to normal (fig 1). The mechanism of these abnormalities of absorption are unclear but may entail delayed gastric emptying, which could readily account for the longer lag time before absorption begins. The delayed rate of absorption could possibly be due to changes in gastrointestinal motility or to oedema of the intestinal wall.
The delayed absorption results in lower peak concentrations of drug at the urinary site of action (fig 1). As such, relatively higher oral doses might have to be given to such patients to attain concentrations necessary to reach the steep portion of the dose-response curve. Due to the unpredictability in an individual patient of the duration of delay in absorption, it is usually easiest to avoid this problem of absorption altogether by simply giving loop diuretics intravenously to patients with $\mathrm{CHF}$ until they attain dry weight. If this strategy is used, oral dosing is then begun when the patient has reached a clinical condition in which optimal absorption for that patient occurs.

\section{Excretion of loop diuretic into the urine} When loop diuretics are given intravenously to patients with CHF who have essentially preserved renal function, both the amount and time course of excretion of the drug into the urine are similar to that which occurs in healthy people (fig 2). ${ }^{5}$ Thus renal clearance is normal. These data mean that normal amounts of loop diuretics are delivered to the urinary site of action. Consequently, abnormalities in response cannot be accounted for by alterations in pharmacokinetics.

If patients with $\mathrm{CHF}$ have diminished renal function, the delivery of loop diuretics into the urine is impaired just as in patients who have primary renal insufficiency (fig 3). ${ }^{5}$ In such patients, doses of loop diuretics need to be altered sufficiently to compensate for the diminished renal function to deliver adequate amounts of drug into the urine. In patients who have adequate renal function, however, no increase in dose is needed. Such patients do not need the same type of increase of dose as is needed in patients with renal insufficiency. As a result, an $80 \mathrm{mg}$ intravenous dose of frusemide, and certainly $120 \mathrm{mg}$, should be sufficient in virtually all patients to get a response if a response is to occur. In turn, to

Absorption of loop diuretics in patients with $\mathrm{CHF}^{5-1}$

\begin{tabular}{ll}
\hline & $\begin{array}{l}\text { Time of peak } \\
\text { concentration (min (SEM)) }\end{array}$ \\
\hline $\begin{array}{l}\text { Frusemide: } \\
\text { Healthy control }\end{array}$ & $108(20)$ \\
CHF: & $242(25)$ \\
Decompensated & $177(21)$ \\
Compensated & \\
Bumetanide: & $72(7)$ \\
Healthy control & No data \\
CHF: & $180(19)$ \\
$\quad$ Decompensated & \\
\hline
\end{tabular}


Figure 1 Absorption profiles of frusemide in two patients with CHF while decompensated and after attaining dry weight. Reproduced with permission.?
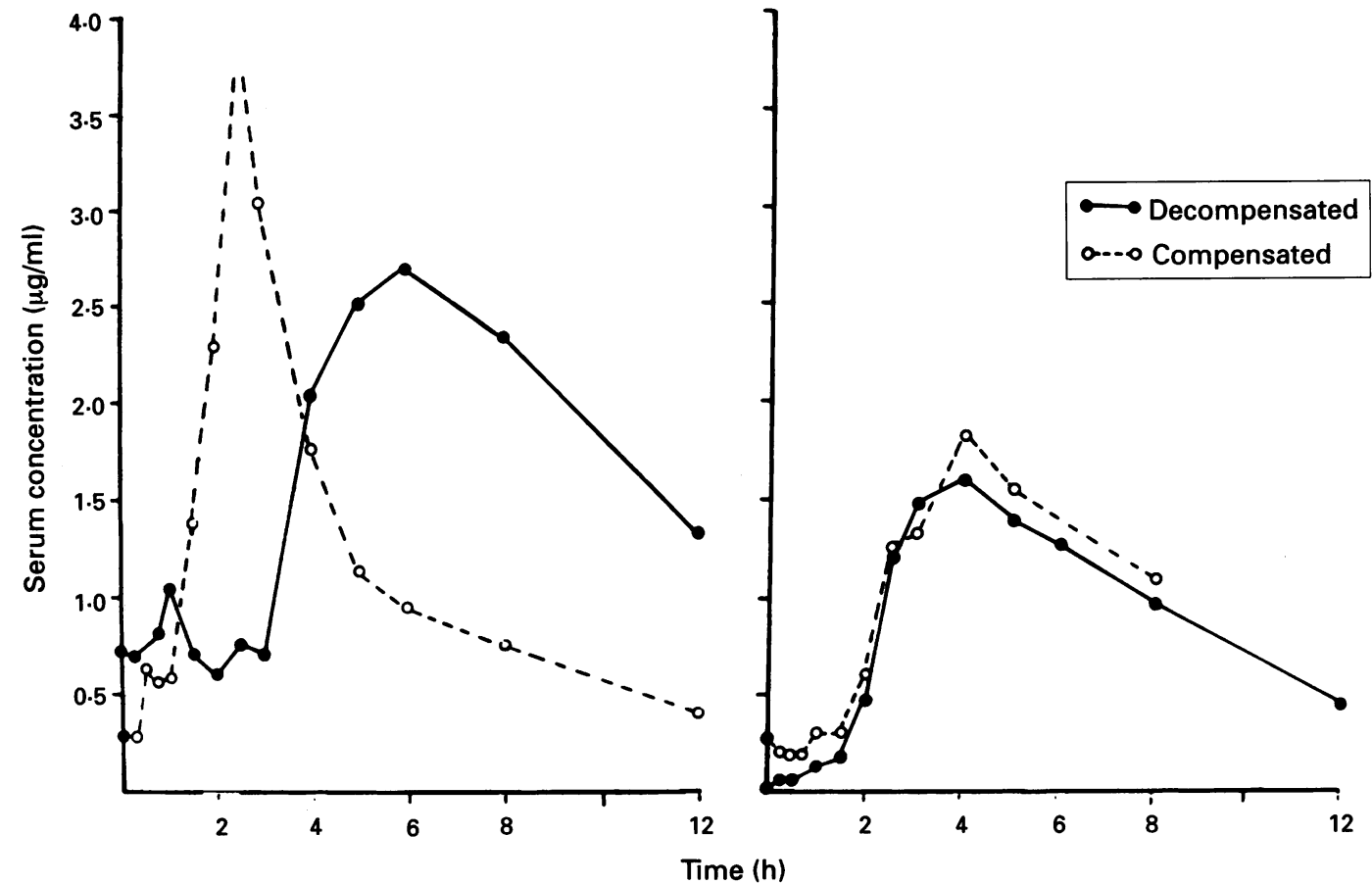

give larger doses than this is unlikely to produce any increase in efficacy and only risks toxicity in patients with CHF. Similar data with bumetanide indicate a ceiling dose to be 2 to $3 \mathrm{mg}$ and for torasemide to be 50 to 100 mg.

These pharmacokinetic data indicate that overcoming resistance to loop diuretics in patients with CHF should not be directed towards increasing doses because adequate amounts of drug are delivered to the urinary sites of action. Instead, treatment needs to improve the pharmacodynamic abnormalities.

Pharmacodynamics of loop diuretics In healthy people, if one assesses the pharma- codynamics of response to a loop diuretic by graphing response as either sodium excretion rate or, more precisely, as fractional excretion of sodium $v$ urinary loop diuretic excretion rate, a sigmoid shaped dose response curve is defined with a maximal fractional excretion of sodium of between $20 \%$ and $25 \% .{ }^{11}$ Sufficient diuretic in the urine to reach this maximum can be achieved with a $40 \mathrm{mg}$ intravenous dose of frusemide, a $1 \mathrm{mg}$ intravenous dose of bumetanide, or 15 to $20 \mathrm{mg}$ of torasemide. That such a high fractional excretion of sodium can occur implies that loop diuretics in maximal doses are capable of completely inhibiting solute reabsorption at the thick ascending limb of the loop of Henle.

In patients with $\mathrm{CHF}$, the dose response
Figure 2 (A) Delivery of frusemide into the urine, and $(B)$ sodium excretion $v$ time in patients with CHF compared with healthy controls.

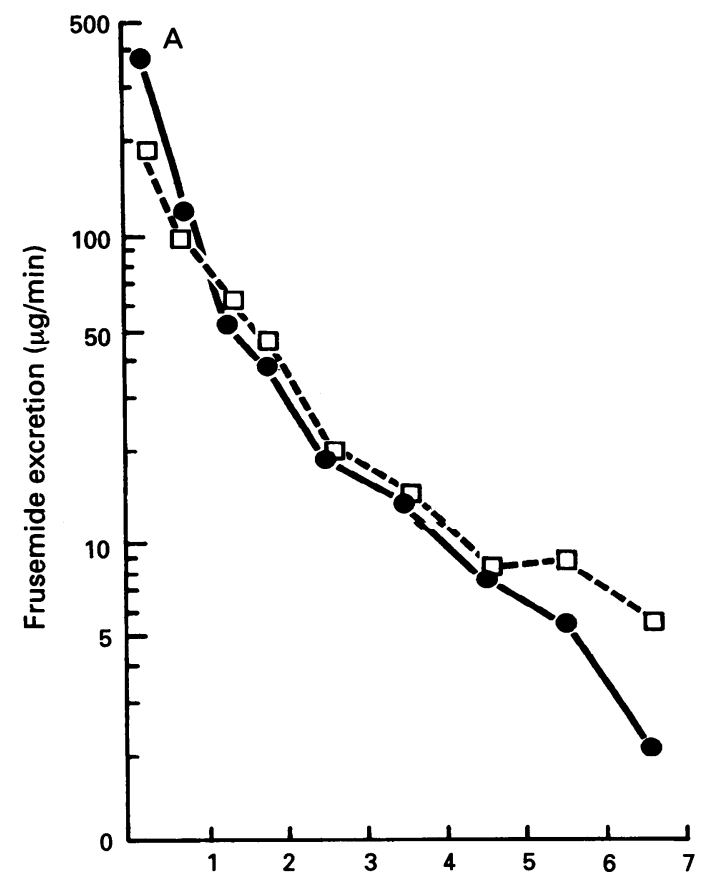

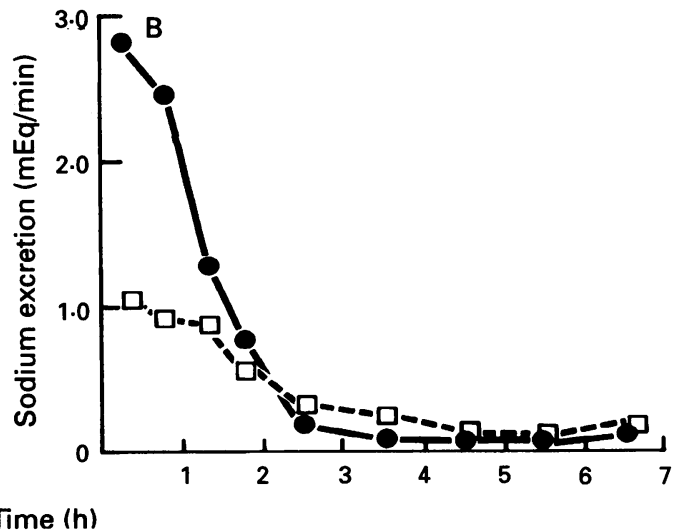




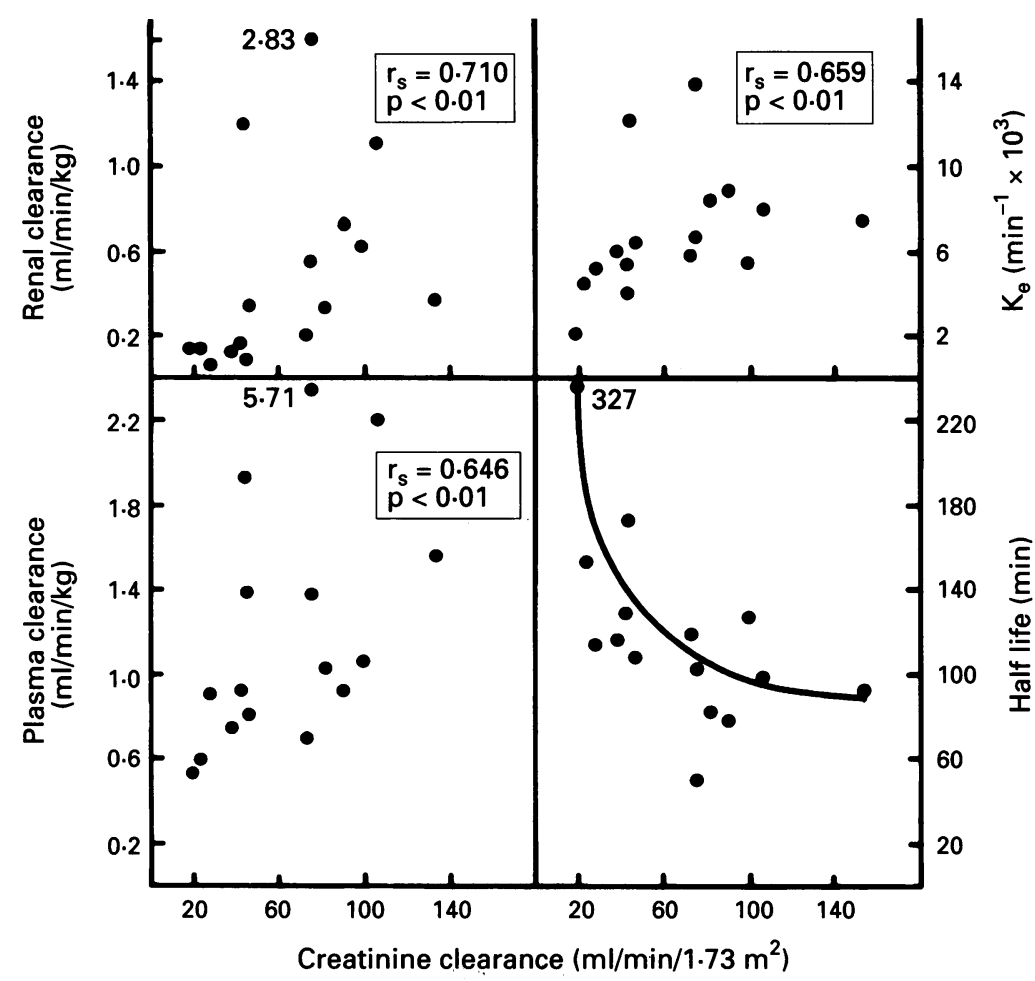

Figure 3 Pharmacokinetics of frusemide in patients with CHF showing the dependence of clearance upon renal function. Reproduced with permission. ${ }^{5}$

curve is shifted downwards and to the right (fig 4). The excretion rate of diuretic causing half maximal response is little changed, but the maximal fractional excretion of sodium that can be obtained is diminished to about $10 \%$ to $15 \%$ on average. ${ }^{12}$ Individual patients may have close to a normal response but some individual patients may show a virtually flat dose response curve indicating that no matter how much diuretic is delivered to the urinary site of action, no response will ensue. If patients have concomitant renal insufficiency, higher diuretic doses are needed to deliver normal amounts of diuretic into the urine. In contrast, as was noted in the previous discussion, if patients have preserved renal function, there is no impairment of delivery of diuretic into the urine. Therefore, patients with $\mathrm{CHF}$ and preserved glomerular filtration rate (GFR) do not need large doses of loop diuretics. The corollary of this finding is that lack of response to doses of 80 to $120 \mathrm{mg}$ of intravenous frusemide or the equivalent of other loop diuretics indicates that there will be no additional response to higher doses and that other therapeutic strategies must be used to get a greater natriuresis. ${ }^{11}$

The mechanism for the abnormal dose response curve in patients with CHF is unknown. Decreased delivery of sodium to the site of action of the loop diuretic or increased reabsorption of solute rejected from the thick ascending limb could account for these changes. ${ }^{11}$ The first of these possibilities could be tested by assessing the effect on response to a loop diuretic of blocking proximal sodium reabsorption. To date no formal studies have assessed the effect of combining acetazolamide with a loop diuretic, although anecdotal experience indicates that acetazolamide can enhance response. Whether this effect is additive or synergistic is unknown. This question has probably not been pursued to a greater extent because acetazolamide could only be given for short periods to patients with $\mathrm{CHF}$, until the metabolic acidosis that occurred secondary to the drug became limiting. Clinicans should remember, however, that in patients who are in critical need of diuresis, it is rational to consider use of acetazolamide in an attempt to cause diuresis when other methods of treatment have failed.

Many publications describe the addition of thiazide diuretics to loop diuretics, the usual result being a synergistic response. ${ }^{13-19}$ In fact,
Figure 4 Shift of the dose-response relation to frusemide in patients with CHF. Reproduced with permission. ${ }^{12}$

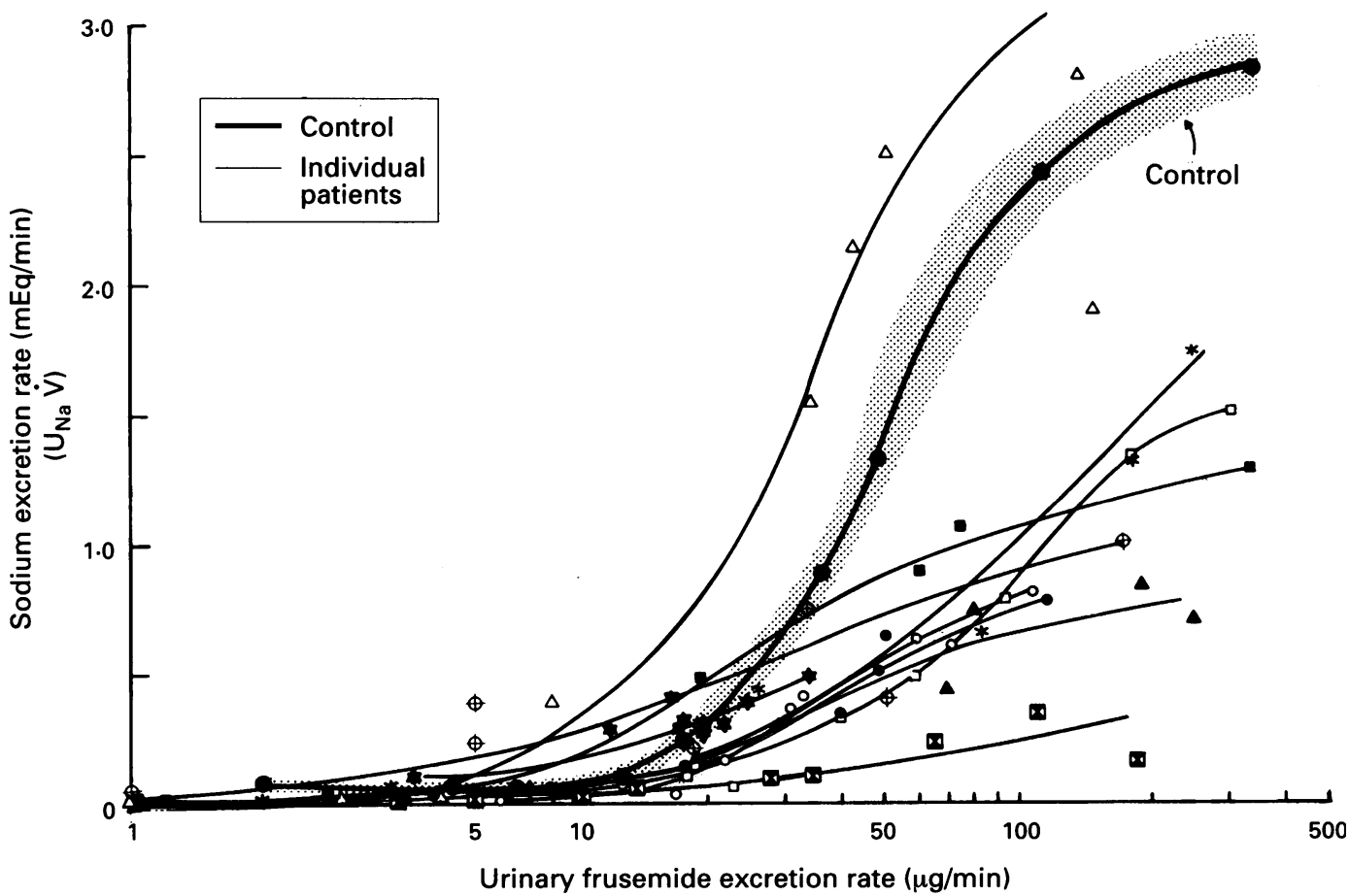


this response can be of such magnitude that volume depletion sufficient to cause cardiovascular collapse has been reported. ${ }^{20}$ Also, blockade of solute reabsorption throughout the thick ascending limb and the distal nephron predictably causes substantial potassium excretion and one must pay particular attention to potassium homeostasis with such combinations of diuretics.

Intriguing recent data clarify the mechanism of the synergistic effect of adding thiazides to loop diuretics. Chronic treatment with loop diuretics floods the distal nephron with sodium and it seems that this serves as a stimulus for hypertrophy of the distal nephrons. ${ }^{21-24}$ This hypertrophy is also associated with increased reabsorptive capacity that in animal models reaches values three to fourfold higher than normal. ${ }^{22}$ Thus, these hypertrophied nephrons seem capable of reabsorbing most, if not all, of the sodium rejected from the thick ascending limb of the loop of Henle and may well account for a decrease in response in patients with CHF chronically treated with loop diuretics. In this setting, addition of a thiazide diuretic blocks sodium reabsorption by these hypertrophied nephrons and results in pronounced natriuresis. ${ }^{25}$ These data account for the rationale of combining thiazide and loop diuretics in patients who have not responded adequately to a loop diuretic alone. In an emergency this strategy can also be used with intravenous diuretics, as chlorothiazide is available as an intravenous preparation.

It also seems that even more distal nephron sites can contribute to the blunted response to a loop diuretic. Because these more distal nephron sites reabsorb sodium in exchange for potassium, one can easily determine whether or not such distal reabsorption is operative in individual patients. Urinary electrolytic determinations in settings of increased distal sodium reabsorption will show low sodium concentrations but raised potassium concentrations. ${ }^{26}{ }^{27}$ In contrast, if the mechanism of decreased response to the loop diuretic is increased sodium reabsorption proximal to these sites, both sodium and potassium concentrations in the urine will be low. If one finds raised urinary potassium concentrations, addition of a potassium retaining diuretic should be considered, the dose of which can be titrated based on excretion of urinary electrolytes. ${ }^{2627}$

In conclusion diuretic resistance in patients with CHF is mostly due to pharmacodynamic factors that seem to be partly related to increased reabsorption of solute in other parts of the nephron. This mechanism explains the rationale for combinations of diuretic drugs in such patients as opposed to increasing doses of loop diuretics themselves. Understanding the mechanisms by which diuretic resistance occurs in patients with $\mathrm{CHF}$ has allowed design of appropriate dosing regimens of these drugs and rational use of combinations of diuretics. The overall result is improved patient care and the ability to produce a desired natriuretic response in nearly all patients.

1 Benet LZ, Greither A, Meister W. Gastrointestinal absorption of drugs in patients with cardiac failure. In: Benet tion of drugs in patients with cardiac failure. In: Benet Washington, DC: American Pharmaceutical Association Washington, DC: American Pharmaceutical Association

2 Benowitz NL, Meister W. Pharmacokinetics in patients with cardiac failure. Clin Pharmacokinet 1976;1: 389-405

3 Odlind BG, Beermann B. Diuretic resistance: reduced bioavailability and effect of oral furosemide. $B M \mathcal{F}$ 1980;280:1577.

4 Greither A, Goldman S, Edelen JS, Benet LZ, Cohn K. Pharmacokinetics of furosemide in patients with congestive heart failure. Pharmacology 1979;19:121-31.

5 Brater DC, Seiwell R, Anderson S, Burdette A, Dehmer GJ, Chennavasin P. Absorption and disposition of furosemide in congestive heart failure. Kidney Int 1982; 22:171-6.

6 Brater DC, Day B, Burdette A, Anderson S. Bumetanide and furosemide in heart failure. Kidney Int 1984;26: 183-9.

7 Vasko MR, Brown-Cartwright D, Knochel JP, Nixon JV, Brater DC. Furosemide absorption altered in decompensated congestive heart failure. Ann Intern Med 1985; 102:314-8.

8 Andreasen F, Mikkelsen E. Distribution, elimination and effect of furosemide in normal subjects and in patients with heart failure. Eur $\mathcal{F}$ Clin Pharmacol 1977;12:15-22.

9 Chaturvedi PR, O'Donnell JP, Nicholas JM, Shoenthal DR, Waters DH, Gwilt PR. Steady state absorption kinetics and pharmacodynamics of furosemide in congestive heart failure. Int $₹$ Clin Pharmacol Ther Toxicol gestive heart failur

10 Fredrick MJ, Pound DC, Hall SD, Brater DC. Furosemide absorption in patients with cirrhosis. Clin Furosemide absorption in patien

11 Brater DC. Resistance to loop diuretics: why it happens and what to do about it. Drugs 1985;30:427-43.

12 Brater DC, Chennavasin P, Seiwell R. Furosemide in patients with heart failure. Shift of the dose-response relationship. Clin Pharmacol Ther 1980;28:182-6.

13 Wollam GL, Tarazi RC, Bravo EL, Dustan HP. Diuretic potency of combined hydrochlorothiazide and furosemide therapy in patients with azotemia. $A m \mathcal{F} \mathrm{Med}$ 1982;72:929-38

14 Sigurd B, Olesen KH, Wennevold A. The supra-additive natriuretic effect addition of bendroflumethiazide and bumetanide in congestive heart failure. Am Heart $f$ 1975;89:163-70.

15 Olesen KH, Sigurd B. The supra-additive natriuretic effect addition of quinethazone or bendroflumethiazide during addition of quinethazone or bendroflumethiazide during long-term treatment with furosemide
tone. Acta Med Scand 1971;190:233-40.

16 Nakahama $H$, Orita $Y$, Yamazaki $M$, Itoh $S$, Okuda $T$, Yamaji A, et al. Pharmacokinetic and pharmacodynamic interactions between furosemide and hydrochlorothiazide in nephrotic patients. Nephron 1988;49:223-7.

17 Gunstone RF, Wing AJ, Shani HGP, Njemo D, Sabuka EMW. Clinical experience with metolazone in fifty-two African patients: synergy with frusemide. Postgrad Med $\mathcal{F}$ 1971;47:789-93.

18 Ram CVS, Reichgott MJ. Treatment of loop-diuretic resistant edema by the addition of metolazone. Current Therapeutic Research 1977;22:686-91.

19 Epstein M, Lepp BA, Hoffman DS, Levinson $R$. Potentiation of furosemide by metolazone in refractory edema. Curr Ther Res 1977;21:656-67.

20 Oster JR, Epstein M, Smoller S. Combined therapy with thiazide-type and loop diuretic agents for resistant thiazide-type and loop diuretic agents for resict
sodium retention. Ann Intern Med 1983;99:405-6.

21 Kaissling B, Stanton BA. Adaptation of distal tubule and collecting duct to increased sodium delivery. I. Ultrastructure. Am f Physiol 1988;255:F1256-68.

22 Stanton BA, Kaissling B. Adaptation of distal tubule and collecting duct to increased $\mathrm{Na}$ delivery. II. $\mathrm{Na}^{+}$and $\mathrm{K}^{+}$ transport. Am $\mathcal{F}$ Physiol 1988;255:F1269-75.

23 Ellison DH, Velàzquez $\mathrm{H}$, Wright FS. Adaptation of the distal convoluted tubule of the rat. $\mathcal{F}$ Clin Invest 1989;83: 113-26.

24 Ellison DH. The physiologic basis of diuretic synergism: its role in treating diuretic resistance. Ann Intern Med 1991;114:886-94.

25 Loon NR, Wilcox CS, Unwin RJ. Mechanism of impaired natriuretic response to furosemide during prolonged natriuretic response to furosemide
therapy. Kidney Int 1989;36:682-9.

26 Alexander WD, Branch RA, Levine DF, Hartog M. The urinary sodium: potassium ratio and response to diuretics urinary sodium: potassium ratio and response to diure

27 van Vliet AA, Donker AJM, Nauta JJP, Verheugt FWA Spironolactone in congestive heart failure refractory to Spironolactone in congestive heart failure refractory to
high-dose loop diuretic and low-dose angiotensin-converting enzyme inhibitor. Am $\mathcal{f}$ Cardiol 1993;71:21A-8A. 